\title{
Multidimensional analysis of monitoring and diagnostic information on the technological process
}

\author{
Aleksandr Pishchukhin ${ }^{1, *}$, and Gulnara Akhmedyanova ${ }^{1}$ \\ ${ }^{1}$ Orenburg State University, 460018 Orenburg, Pobedy avenue 13, Russia
}

\begin{abstract}
The study divides the analysis of monitoring and diagnostic information, leaving for monitoring the parameters of the stationary process, and for diagnostics - various transient regimes in the technological process. This, in turn, divides the algorithms of information processing into control of the output from the given boundaries for stationary parameters and the classification and prediction for dynamically changing parameters in a multidimensional space. In view of the large number of monitoring and diagnostic information, as well as due to different algorithms for processing it in an appropriate information system, it is necessary to apply multi-dimensional analysis methods. As diagnostic influences, various jumplike changes of a "natural character" are used, and the state of the equipment allows judging the apparatus of the influence functions. The abrupt changes in the technological process are reflected in the change in its parameters. The reaction to them is weakened as they are removed in accordance with the influence functions. The values of the parameters at the moment of the reaction define a point in the multidimensional parameter space and allow one to relate the state to one or another standard, and to relate the corresponding management algorithm to the standard. The experimental model includes five links simulating the operations of the technological process, a pulsed signal source simulating a step change and five links of propagation delay simulating the duration of operations. The results confirmed theoretical conclusions about the influence functions.
\end{abstract}

\section{Introduction}

The normally flowing technological process is characterized by the values of the parameters, information on each of which can be useful from the point of view of the reliability of the functioning of the technological equipment or the quality of the manufactured products. At the same time, the information received at the current time (monitoring) can be used to stop the process in the event of deviations of the parameter values from the established thresholds, but it is of little use even for short-term forecasting. From this point of view, diagnostic information is more promising, that is information on

*Corresponding author: pishchukhin55@mail.ru 
the reaction of technological equipment to test impacts. However, to obtain it, it is necessary to stop the technological process. At the same time, in the course of the technological process, sudden changes occur - changing or reinstalling the tool, changing the properties of the workpieces, cutting in and out of the tool, etc. For these changes, it is possible to predict the condition of the equipment and prepare a plan for interventions in the future technological process with the allocation of the necessary resources. A large number of received information, as well as the lack of appropriate algorithms for its processing, make the research presented in this direction relevant.

\section{Theory}

Monitoring and diagnostic systems are an important part of automated technological process control systems (ACS TP). The introduction of such systems, as noted in [1], "acquires special significance, since it allows to ensure the effective operation of enterprises in given modes, improve the quality of products, ensure trouble-free operation and environmental safety of production, increase labor productivity." Although the primary task of such systems is to control the parameters of the technological process, they still have the accumulation of information, the analysis of the available experience, even the prediction of certain events, for example, repair work [2]. It tools demonstrate good applicability for successfully solving problems in the production of iron, iron ore sintering and the hot strip rolling process with regard to process control or product quality [3]. The development and integration of monitoring, diagnostic and forecasting technologies (collectively known as PHM) can improve maintenance and control strategies in production operations [4]. PHM technologies are actively studied for the purpose of application in robotics for the promotion of innovative technologies for recognition and prediction [5]. In our country, researchers also develop the structures of process monitoring systems and algorithms for analyzing the dynamics of changes in output variables and the parameters of the state of the technological system $[6,7,8,9]$.

From the point of view of ACS TP, technological processes are divided into two large classes: continuous and discrete. "Continuous is a process in which the final product is developed as long as raw materials, energy, catalysts, control actions are brought in" [10]. Such processes can include, for example, the processes of the chemical or food industry. "Discrete is a technological process in which the final product is developed at specific intervals, and this process can be stopped, and continue with any technological operation without reducing the specified level of quality" [10]. You can name such examples as: the functioning of automatic lines, the process of assembling products on a conveyor, the testing of finished products, etc.

In the stationary mode, when there is no time dependence, the description of the technological processes can be independent of the allocated classes, since the monitoring points are discretely distributed according to the sequence of operations $-i=1, \ldots, m$. In the simplest form, the dependencies can be linear mappings.

$$
y_{i}=\sum_{j=1}^{n} a_{i j} x_{j}+b_{i}
$$

where $x_{j}$ - are the input parameters of the operation, for example, the aggregate of the processed product size or the set of parameters of the processed medium $(j=1, \ldots, n)$, and $y_{i}$ are the output parameters, $a_{i j}, b_{i}$ are the transformation coefficients of the processed product or medium. We note that in the course of the technological process, the changes in the output parameters must obey the principle of compressive mappings, while the compressibility conditions [11]: 


$$
\sum_{j=1}^{n}\left|a_{i j}\right| \leq \alpha<1
$$

In this case, the process converges to the so-called fixed point, the coordinates of which satisfy equation (1) and as which the product quality index at the output of the technological process can be chosen.

Diagnostics is connected with the supply of test signals, in this study such signals are abrupt changes in the technological process, connected with the analysis of the process reaction in time. Therefore, in this case the description of continuous processes and discrete ones has a different mathematical apparatus.

Continuous processes are associated in practice with heat fluxes and temperature changes, changes in concentrations at different points in the medium, filtration and similar physical processes, and can conveniently be described by a parabolic partial differential equation [12], which reflects the so-called relaxation processes. It describes the behavior of an environment in which energy or matter does not arise and does not disappear, in a more general sense, there is no source for changing the parameter $y_{i}$. If it is necessary to actively influence this environment in technological operations, then it appears on the right side of this equation in the form of concentrated or distributed sources.

$$
\frac{\partial y_{i}}{\partial t}-\alpha^{2} \frac{\partial^{2} y_{i}}{\partial x^{2}}+\beta \frac{\partial y_{i}}{\partial x}+\gamma y_{i}=0
$$

where $y_{i}$ is the controlled parameter, $\alpha$ is the diffusion coefficient, $\beta$ is the drift coefficient, $\gamma$ is the proportionality coefficient, $x$ is the distance from the beginning of the process, $t$ is the time.

The solution of this equation is determined through the influence function or the Green's function (the function of a point source)

$$
G(x, \xi, t)=\frac{1}{2 \sqrt{\pi a^{2} t}} e^{-\frac{(x-\xi)^{2}}{4 a^{2} t}},
$$

where $\xi$ is the position of the point source.

Control in the technological process is carried out, as mentioned above, through the right side of the equation (3).

$$
\frac{\partial y_{i}}{\partial t}-a^{2} \frac{\partial^{2} y_{i}}{\partial x^{2}}+\beta \frac{\partial y_{i}}{\partial x}+y_{i}=u(x, t) .
$$

In this case the solution with zero initial conditions looks like a convolution

$$
y_{i}(x, t)=\int_{0}^{t} \int_{-\infty}^{\infty} G(x, \xi, t-\tau) u(\xi, \tau) d \xi d \tau
$$

The two types of description - on the basis of the principle of contracting mappings and the partial differential equation allow us to conclude that the magnitude of the response of the controlled parameters decreases with distance from the beginning of the technological process.

Each operation of the discrete technological process involves the transfer of the product to a new level of values of the operation parameters. We shall describe this translation by an aperiodic link on the basis of equation 


$$
T \frac{d y_{i}}{d t}+y_{i}=k x_{i}
$$

where $T, k$ are the constants of the aperiodic link (operations), $x_{i}$ are the input parameters of the $i$-th operation, $y_{i}$ are the output parameters. The advantage of this description, apart from the simplicity of the equation, is the exponential (non-oscillatory) response to the input effect, as is the case with the regular operation. If during the operation energy is stored or released (for example, due to increased gaps in the equipment and the need for repair), the description is reduced to the equation of the oscillatory link

$$
T^{2} \frac{d^{2} y_{i}}{d t^{2}}+2 T D \frac{d y_{i}}{d t}+y_{i}=k x_{i}
$$

However, the decrease in the response to a stepwise effect on any intermediate operation with such a description should be investigated additionally.

\section{Model and methods}

To ensure that the quality of products meets specified requirements, the variability of the process benchmarks should be within certain forecasted limits.

In view of the large number of monitoring and diagnostic information, as well as various processing algorithms, multivariate analysis methods are applicable here. In this case, each monitored parameter can be assigned its own coordinate axis, and the values obtained can only be filtered and smoothed for the analysis of only the stationary mode. The control algorithm in this case is very simple

$$
y_{i m}<y_{i}<y_{i v}, i=1, \ldots, n,
$$

where $y_{i m}, y_{i v}$ - respectively, the lower and upper values of the $i$-th parameter, $n$ - the total number of monitored parameters.

Diagnostic information is processed only in the case of abrupt changes in the course of the technological process: changing and reinstalling the tool, changing the properties of blanks, this or that abnormal situation. At the same time, the degree of influence of the event is evaluated depending on the distance from the zone of abrupt change. If this influence turns out to be close to the described by equations (2), (6) or (7), localization and fault recognition algorithms are connected. If this influence deviates from the standard one, it is necessary to connect additional algorithms connected with the analysis and forecast of future contingencies at other points of the technological process, different from the place where the jump occurred, but the first ones that showed a deviation from the standard behavior during the process [13].

In a multidimensional space, the standard reaction to an abrupt change in the values of the parameters that play the role of coordinates forms a point - the standard of the class. Near this standard a cloud of points of images of a given class is formed. Accordingly, as a sign of the entry of the image under investigation into this class, we can assume the condition of falling into the sphere of a given radius $r$ with center at the reference point of the class

$$
\sqrt{\sum_{i=1}^{n}\left(y_{i}-y_{i}\right)^{2}} \leq r
$$


where $y_{i}$ are the coordinates determined by the real response, $y_{i}{ }^{3}$ are the coordinates calculated by the model and taken as the reference.

Each class is associated with an algorithm for responding to an event that occurred, both for immediate actions, and for planning the allocation of certain resources, changing load schedules, product sizes, and so on. [14]

Let us investigate the described phenomena on the model shown in Fig. 1.

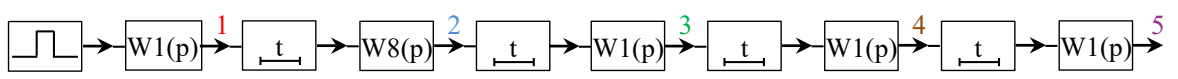

Fig. 1. Schematic diagram of the technological process.

The model includes five links simulating the operation of the technological process, a pulsed signal source simulating a step change and five links of signal propagation delay simulating the duration of operations.

The following notation is used in the diagram: W1 (p) is the transfer function of the aperiodic link, W8 (p) is the transfer function of the vibrational link simulating nonstandard dynamic behavior.

\section{Results and discussion}

In the absence of an oscillatory link, the circuit shows a standard deviation, as shown in Figure 2

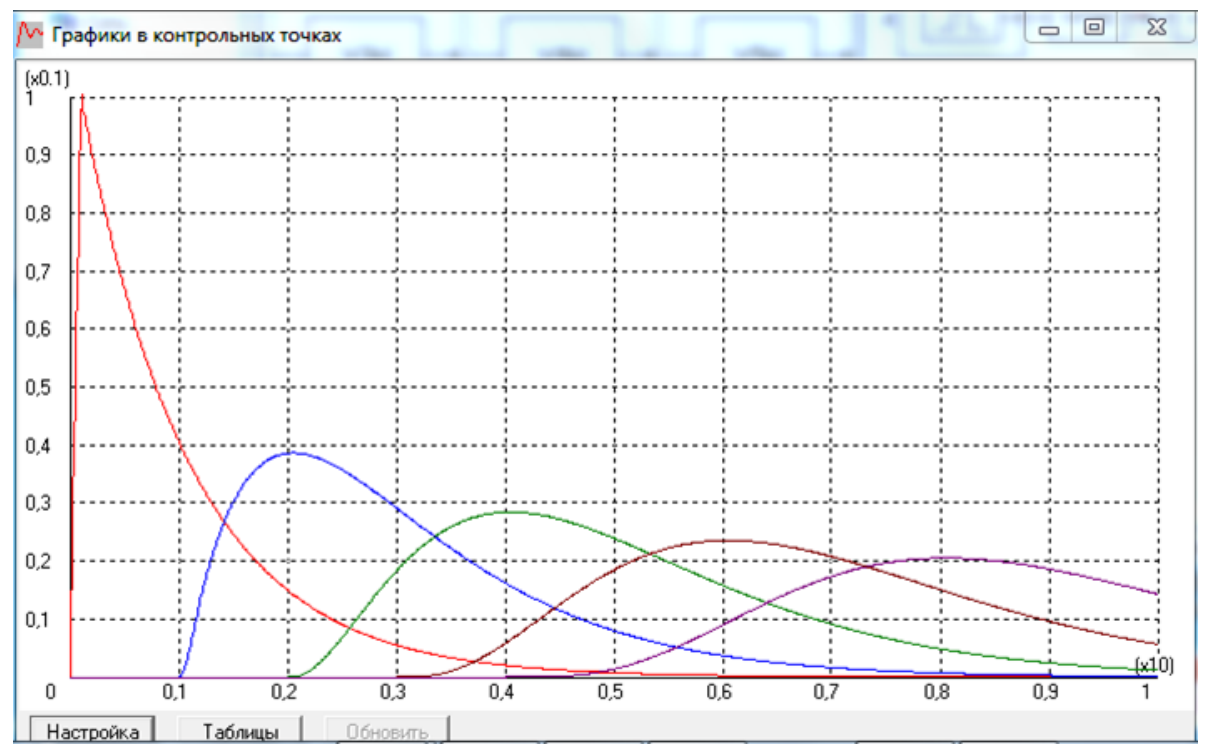

Fig. 2. Standard reaction of the process model to an abrupt change

As we can see, the reaction to the jump in the beginning of the technological process weakens as it moves along the way. And this weakening is most strongly at the beginning (between the first and second links), then the decline decreases, the further, the more.

If in the process chain there is equipment on the verge of failure in the form of an oscillatory link, the model demonstrates the non-standard behavior shown in Figure 3. 
Moreover, after the "defective" link the reaction again becomes standard, that is, it "freezes" as it is removed.

\section{Conclusion}

Thus, multidimensional analysis of monitoring and diagnostic information about the technological process allows you to control parameters, and predict the future behavior of equipment. At the same time, various jumplike changes are used as diagnostic effects, and the apparatus of influence functions can be judged on the state of the equipment. Jumps change the parameters of the technological process. The reaction to them is weakened as they are removed in accordance with the influence functions. The values of the parameters at the time of the reaction set the point in the multidimensional parameter space and allow one to relate the state to one or another standard, and to relate the corresponding management algorithm to the standard.

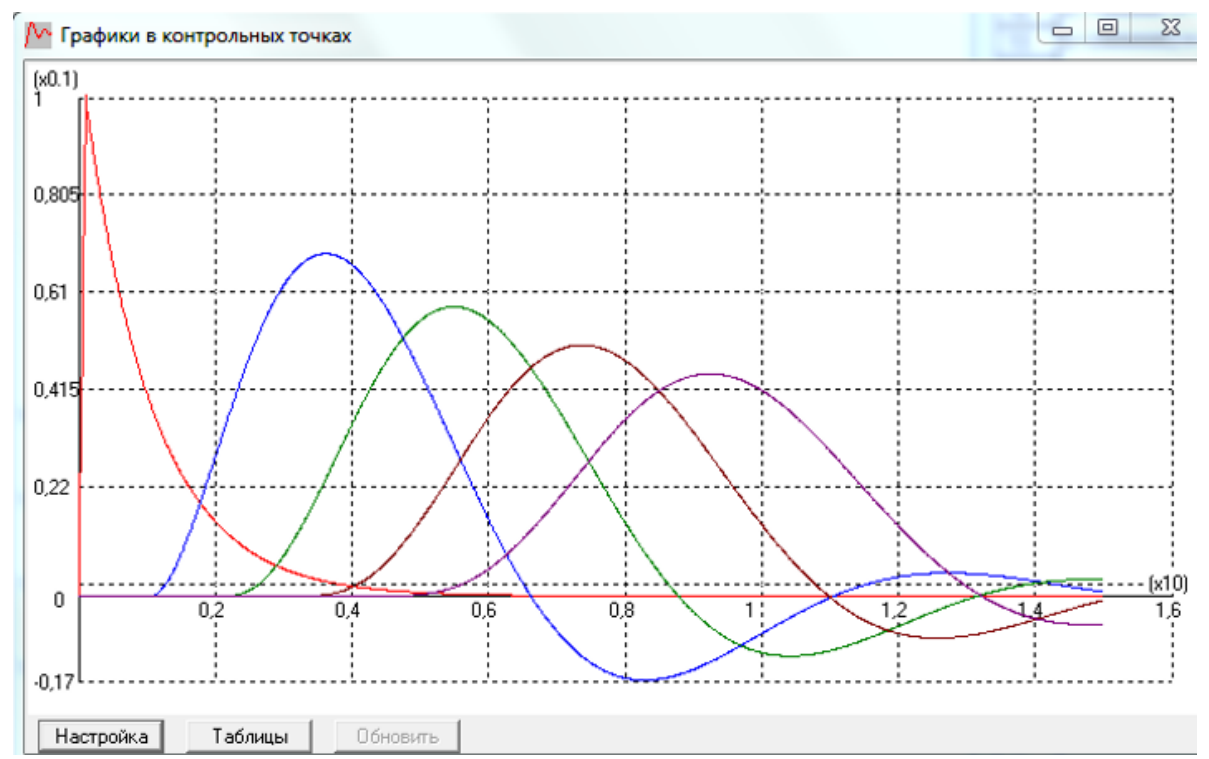

Fig. 3. Non-standard reaction of the process model to an abrupt change.

\section{References}

1. E.S. Okhotnikov, Systems for monitoring the technological processes of oil and gas producing enterprises: classification and mathematical modeling Oil and gas business, (2006). (Access mode: http://www.ogbus.ru)

2. D. Shvetsov, Intellectual systems of data storage in the automated process control system [Electronic resource]. URL: http://www.cta.ru/cms/f/441322.pdf (reference date: $11 / 04 / 2018)$.

3. C. C. Wang, C. H. Ko, J. S. Shiau, W. Long Development of Monitoring and Diagnosis Technologies for Steel-Making Process in CSC, Optomechatronic Technologies (ISOT), International Symposium on 5-7 Nov. (2014).-Seattle, WA, USA. Access mode: https://ieeexplore.ieee.org/document/7119397/ 
4. B. A. Weiss, M. Helu, G. Vogl, G. Qiao Use Case Development to Advance Monitoring, Diagnostics, and Prognostics in Manufacturing Operations, IFACPapersOnLine 49-31, p. 13-18 (2016)

5. G. Qiao, B.A. Weiss. Weiss Advance Measurement Science to Assessments, Diagnostics, and Prognostics for Manufacturing Robotics Int J Progn Health Manag. 7 (2016); (Spec Iss on Smart Manufacturing PHM): 013.

6. E.E. Yagyaev, Methodical support of the monitoring system for the technological process of fine grinding, Proceedings of the Russian State Pedagogical University, p.30-36. (2015)

7. D.O. Pochufarov, Identification and analysis of technological processes of objects (enterprises) as a measure of reducing losses in idle times / D.O. Pochufarov, P.V. Lebedkin, V.V. Moiseev, A.V. Ketov // Youth and Science: A collection of materials of the VII All-Russian Scientific and Technical Conference of Students, PhD students and young scientists dedicated to the 50th anniversary of the first manned flight into space [Electronic resource], (Krasnoyarsk: Siberian Federal University 2011). - Access mode: http://conf.sfu-kras.ru/sites/mn2011/section19.htm

8. A.M. Pishchukhin, G.F. Akhmedyanova G.F. Automation and technology - two aspects of the technical equipment of production, Bulletin of the OSU. 9 184, pp. 33-36 (2015)

9. A.M. Pishchukhin, G. F. Akhmedyanova The Metasystem Interconnection of Automated Workplaces in Enterprise Management, Collected materials of the International Scientific Conference dedicated to the 60-th anniversary of the Orenburg State University "Science and Education: Fundamental Foundations, Technologies, Innovations", (Orenburg: OSU, 2015).- pp. 235-239.

10. Classification of technological processes ASU TP. Access mode: https://automationsystem.ru/main/11-asutp/asu-tp/45-40-klassifikacziya-texnologicheskix-proczessovasu-tp.html

11. A.N. Kolmogorov, S.V. Fomin, Elements of the theory of functions and functional analysis (M .: Nauka, 1981)

12. A.N. Tikhonov, A.A. Samarskii, Equations of mathematical physics (Moscow: Nauka, 1977)

13. A.M. Pishchukhin, Increasing the survivability of GPS based on metasystem replacements, Izvestiya Samara Scientific Center of the Russian Academy of Sciences. 18, 4-1 (2016)

14. A.M. Pishchukhin Consistency of the components of the system and methods for its achievement, Bulletin of the Orenburg State University 1 (1999) 